\title{
Strategy and temperature affect of the charging of lithium-ion battery management system XIONG Yi
}

(Hunan Vocational College of Railway Technology, ZhuZhou Hunan 412001)

\begin{abstract}
Keywords: Lithium-ion battery; battery management system; a charging policy; temperature.
\end{abstract}
\begin{abstract}
This paper mainly research the relationship between the lithium-ion battery management systems and charging policies and temperature. In the introduction of lithium-ion battery characteristics of the premise, the lithium-ion battery protection methods are described, based on a lithium-ion battery protection method in detail its charging policies and to study the effect of temperature on charging policies, compared with good interpretation of the lithium-ion rechargeable battery management system strategy selection and temperature control importance of the lithium-ion battery protection, providing a theoretical basis and ideas for research and specific battery management system.
\end{abstract}

\section{Introduction}

Lithium-ion battery is a lithium metal oxide alloy anode material, graphite as the cathode material, the use of non-electrolyte rechargeable battery. As lithium battery materials it has many advantages, but also has a very active features highly flammable, and burning caused by high temperatures and the volume expansion tends to cause disastrous consequences, therefore, in addition to the lithium-ion battery itself in the production, storage, transport, the operation to enforce strict specifications, the management and selection policy to charge and discharge its important, in addition, the effect of temperature in the battery management system should not be ignored.

\section{2. lithium-ion battery strategy}

\subsection{Relationship between battery capacity and cycle life and charging cut-off voltage}

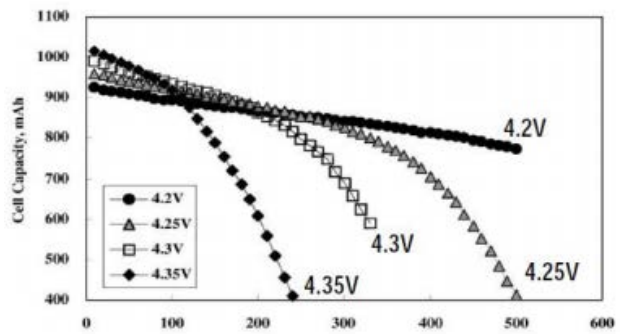

Fig. 1 Lithium-ion battery capacity and cycle life and charging cut-off voltage.

Fig. 1 is a capacity of 950mAh lithium-ion battery capacity and cycle life and charging cut-off voltage. Figure.As can be seen from the figure, alone 4.35V lithium-ion battery protection circuit provides a charge-cutoff voltage, then it's initial capacity is relatively large, but very short cycle life, capacity drops fast.

\subsection{Relationship between battery capacity and cycle life and discharge current}

Fig. 2 lithium-ion battery capacity and cycle life and discharge current diagram, can be seen from, for $900 \mathrm{mAh}$ lithium-ion battery, 1.0C-rate charging capacity after 500 cyclesof about 78 $0 \mathrm{mAh}$, single terms of $2.0 \mathrm{C}$ rate

For charging capacity after 500 cycles of about $500 \mathrm{mAh}$, the trend can be seen from the following graph, with the upgrading of the charging rate, the capacity fading rate is substantially increasing exponentially. 


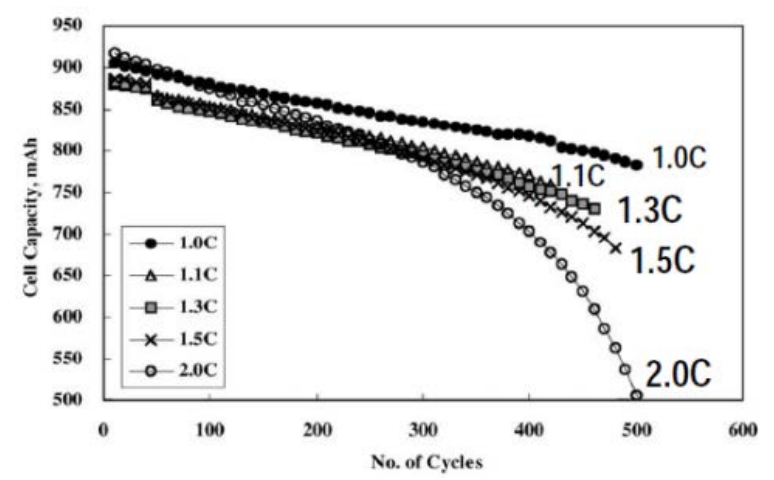

Fig.2 lithium-ion battery capacity and cycle life and discharge current relationship diagram

\subsection{Lithium-ion batteries Policy}

During charging, the charger under external electric field is applied, $\mathrm{Li}+$ released from the positive to the negative electrode into the electrolyte in $\mathrm{LiCoO} 2$ move, turn into negative electrode graphite, and in that form $\mathrm{LiC}$ compound. If the charging too fast, it will make $\mathrm{Li}+$ late into the negative electrode grid in the vicinity of the negative electrode electrolyte will gather $\mathrm{Li}+$, which is close to the negative $\mathrm{Li}+$ is likely to capture an electron from the negative to metallic Li. Will generate continuous metallic lithium anode piled nearby, grew into dendritic crystals, commonly known as dendrites, another situation, with the full extent of the negative increasingly high, LiC lattice leaving less and less space, from the positive movement over the $\mathrm{Li}+$ chance of finding less and less space required more time, if the charging rate does not change, the same may be formed in the surface of the negative local $\mathrm{Li}+$ accumulation, therefore, in the second half of the charge must gradually reduce the charge current, dendrites grow up eventually pierce the separator between the positive and negative electrodes, a short circuit, it is conceivable, faster charging more dangerous, the more dangerous the higher cut-off voltage charging, charging time is longer more dangerous 。

Based on the understanding of the characteristics of lithium-ion batteries, the industry has formed a three-stage strategy for the lithium-ion battery charging: pre-charge, constant current charging, constant voltage charging. The significance of the pre-charge state of the battery is adjusted so that it can enter the state of large current charging, constant current charging of the electric energy is stored in the battery in a fast, constant adjustment phase is the last stage, which allows the battery maximize capacity, but the process is carried out in full accordance with its own battery needed, unlike the constant current charging as the battery has a strong electric field force, and any act contrary to the battery characteristics, especially over the battery capacities are too large current or exceed the battery voltage overshoot to the battery life operations will have a huge impact, so any good management program must be carried out in accordance with strict design specifications.

Three-stage strategy view of a lithium-ion battery charging time is shown in Fig.3.

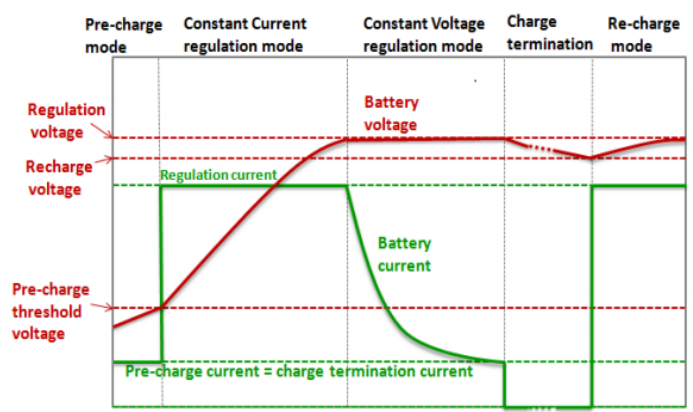

Fig.3 Three-stage strategy view of a lithium-ion battery charging

Precharge occurs in the battery voltage is relatively low for most lithium-ion battery, this voltage is generally defined in $2.9 \mathrm{~V}-3 \mathrm{~V}$ or less, when the allowable charging current $\mathrm{C} / 10$ or less. Constant charging current is generally set at about $1 \mathrm{C}$, at constant voltage charging phase, the charging current will gradually decrease the value of the current must be reduced (usually C / 10) later, when the battery is fully charged, the charging process will be closed, Figure 4 displays the last stage is 
called supplementary stage, it is actually a combination of a constant current stage and constant voltage stage, which is to compensate battery self-discharge capacity and other consumption of the load connected to it caused by falling remedies, this is to ensure that the battery and charging device separate as much as possible is always in full charged state.

\section{In which the temperature affect battery charging policy}

The temperature of the battery which has a significant impact on the charging policy. Due to the different characteristics of materials at different temperatures of the battery, the battery capacity, the appropriate charging voltage will change dramatically.

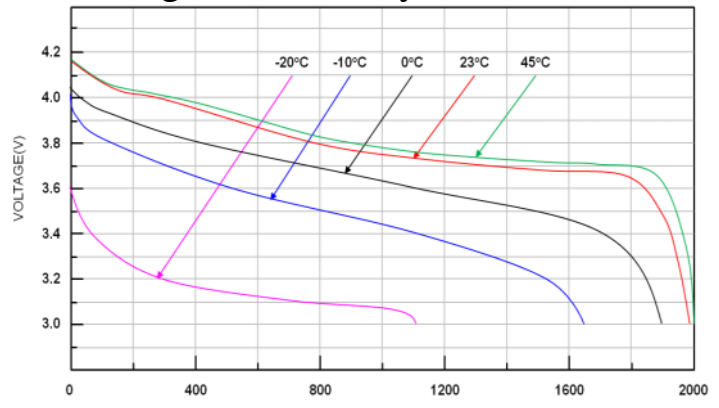

Fig.4 Effect of temperature on the lithium-ion battery capacity and voltage

Typically, the temperature is too low or too high temperature should prohibit the lithium-ion battery, battery management systems generally use RT9503A to achieve, its application circuit shown in Fig.5. The dotted line together is at a variable resistor and battery together, this variable resistor is a thermistor, put it and the battery discharge in order to show the measured temperature of the battery itself, in practical work, RT9503A the TS interior flowing current of 102uA, and the current flows through the thermistor in the TS will end after the formation of a voltage, if the voltage between $0.5 \mathrm{~V}-2.5 \mathrm{~V}$, is considered a temperature in the range of rechargeable, and once the voltage exceeds this range, it said they did not fit, the charging process will be terminated immediately, in order to protect the battery charge will not be due to improper damaged.

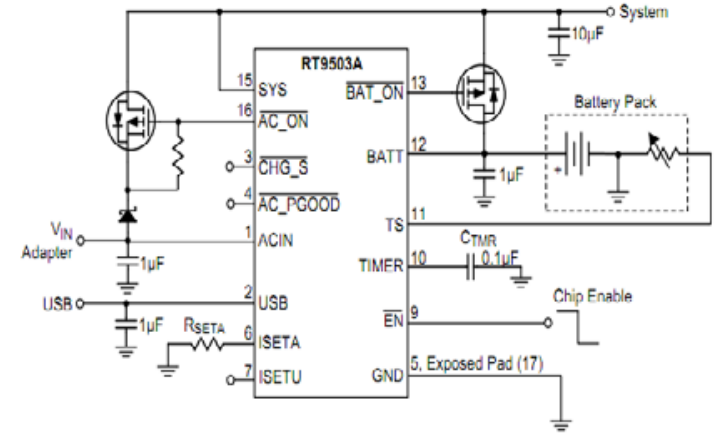

Fig. 5 RT9503A application circuit diagram

Secondly, according to the internal circuit diagram RT9528, special offers TEMPIN, while TEMPIN signal input a set of comparators, these comparators compare the results will be given according to the voltage level, representing the hot, hot, cold, cold. The controller will adjust the parameters and specifications in accordance with the judgment result of these pre-established.

Charging voltage changes Fig. 6 and Fig.7 shows a specific temperature from room temperature were cold, too cold and then changed when the charging current and temperature changes in the ambient temperature gets hot, then changed to hot. 


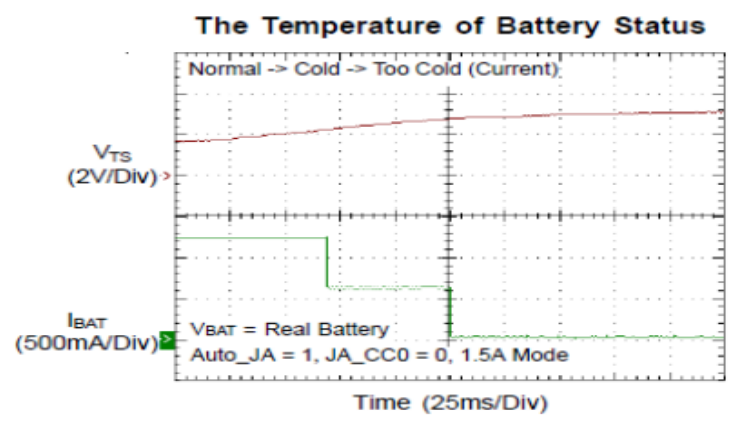

Fig. 6 cooling from room temperature, the charging current is then changed to cold changes

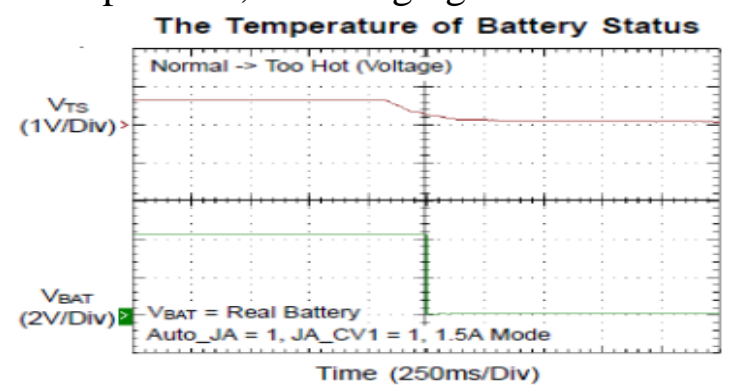

Fig.7 Charging temperature from room temperature to heat, and then changed to a hot voltage changes Figure

In addition, some designed by external control circuitry or communications interface command to change the charging current and voltage. While some devices are only the most basic constant current and constant voltage output, set all the parts are open to the hardware designer, which build a comprehensive battery management system, it has a positive meaning.

\section{Summary and Conclusions}

This paper describes a lithium ion battery characteristics under the premise of the lithium-ion battery protection methods are described, based on a lithium-ion battery protection method, elaborated its three-stage charging policies and to study the temperature of the charge influence policy, better explain the importance of controlling the lithium-ion rechargeable battery management system strategy selection and temperature lithium-ion battery protection. In addition, under the existing IC conditions, open hardware setting section privilege also to develop lithium-ion battery management system provides the direction of thinking.

\section{References:}

[1] Hu Guangxia. Lithium-ion battery charging and discharging process [D]. Chinese Academy graduate master's degree thesis. 2012.

[2] Song Bin thermoelectric lithium-ion battery chemistry and computing and simulation electrode materials [D]. Central South University doctoral dissertation in 2013.

[3] Research and design wave music. Lithium-ion battery integrated test system [D]. Harbin Institute of Technology master's degree thesis. 2006.

[4] Liu Yanbo. Improving high-temperature cycling stability of lithium-ion battery research [D]. Shanghai Jiaotong University master's degree thesis. 2013.

[5] Liu Quanbing. Preparation and Properties of Lithium-ion battery cathode materials research [D]. South China University of Technology doctoral thesis. 2012.

[6] History Research turn on the lithium-ion battery thermal stress analysis and the thickness variation of [D]. Beijing Nonferrous Metal Research Institute master's thesis in 2014. 